\title{
OPTICALLY INDUCED GAPS IN DISORDERED SEMICONDUCTORS
}

\author{
A. KALVOVÁ ${ }^{a}$ AND B. Velick'́ $Y^{a, b}$ \\ ${ }^{a}$ Inst. of Phys., Czech. Acad. Sci., Na Slovance 2, 18040 Praha 8, Czechia \\ ${ }^{b}$ Faculty of Math. and Phys., Charles University, Ke Karlovu 5, 12116 Praha 2, Czechia
}

On the example of an explicitly solvable model of a semiconductor with alloy disorder in the conduction band, it is shown that a slowly varying exciting light pulse can be treated in an adiabatic approximation, that is, the self-energy of an electron can be taken as a continuously evolving series of snapshots of self-energies corresponding to a steady illumination with the instantaneous value of the light strength.

PACS numbers: 78.47.+p, 71.25.-s

\section{Introduction}

A realistic theory of fast optical transients in disordered semiconductors should be suitable for determining the response to optical pulses with intensities varying in a wide range and of fairly general shapes. The transport type theories are often not sufficient, as the transients are, as a rule, non-thermalized and quantum coherence between the exciting field and the internal dynamics of the system occurs. A fully quantum theory [1] was proposed and further developed in [2] and [3]. While this work illuminated basic notions like the so-called generalized Kadanoff-Baym ansatz, an explicit solution was limited to the sudden onset of the light intensity. This limitation was related to the technique used, namely the double time Green functions (GF) of the Kadanoff-Baym type.

This communication presents first resul ts obtained for pulses of more general shapes. We limit our task in two directions. We find only the retarded propagators under such transient conditions. Already this is quite involved. It is then important to study the limits. In contrast to the abrupt onset treated in [2], we study here the opposite, "adiabatic" limit of the pulse.

After the preliminaries of Sec. 2, we propose in Sec. 3 to treat the electron propagation as a sequence of time evolutions under steady illumination with a slowly varying strength. In Sec. 4 we concentrate on the field and disorder dependent optically induced gap in the adiabatic self-energy. 


\section{Model Hamiltonian and the coherent potential approximation propagators}

The Hamiltonian describing the resonant action of a very strong electromagnetic field on a two-band semiconductor with substitutional disorder in the conduction band has the following form in the Schrödinger representation:

$$
H_{\mathrm{S}}(t)=W_{\mathrm{v}}+W_{\mathrm{c}}+V_{\mathrm{c}}+U(t) .
$$

IIere the band IIamiltonians $W_{b}=P_{b} W_{b} P_{b}, b=c$, v, are diagonal in the Bloch basis and the band projectors $P_{\mathrm{v}}, P_{\mathrm{c}}$ are non-random. The random potential is site diagonal

$$
\begin{aligned}
V_{\mathrm{c}} & =P_{\mathrm{c}} V_{\mathrm{c}} P_{\mathrm{c}}=\sum_{i}|c i\rangle \epsilon_{i}\langle c i|=\left\langle V_{\mathrm{c}}\right\rangle+\sum_{i} \mathcal{D}_{\mathrm{c} i} \\
& =\langle\epsilon\rangle P_{\mathrm{c}}+\sum_{i}|c i\rangle \underbrace{\left(\epsilon_{i}-\langle\epsilon\rangle\right)}_{d_{i}}\langle c i|
\end{aligned}
$$

and there is no correlation between $d_{i}$ for different sites. Finally, $U(t)$ is a non-random dipole interaction with a quasimonochromatic light wave with frequency $\omega$ treated in the rotating wave approximation (RWA). A unitary transformation attributed to Galitskii [4], $O(t)=P_{\mathrm{c}}+P_{\mathrm{v}} \mathrm{e}^{\mathrm{i} \omega t}$, brings the IIamiltonian (1) to

$$
\begin{gathered}
H_{\mathrm{S}} \rightarrow H(t)=O H_{\mathrm{S}} O^{+}=W_{\mathrm{c}}+V_{\mathrm{c}}+\left(W_{\mathrm{v}}+\hbar \omega P_{\mathrm{v}}\right)-e \mathcal{E}(t)\left(x_{\mathrm{cv}}+x_{\mathrm{vc}}\right) \\
\equiv W_{\mathrm{c}}+V_{\mathrm{c}}+\widetilde{W}_{\mathrm{v}}+\tilde{U}(t),
\end{gathered}
$$

where $\mathcal{E}(t)$ is the electric field amplitude, $x_{\mathrm{cv}}$ - the dipole transition matrix. After the transformation, the Hamiltonian has the valence band shifted by $\hbar \omega$ to satisfy the weak field resonance condition for the optical transitions, which appear now as an effective band hybridization with a time-varying hybridization strength. This is the only explicit time dependence in the IIamiltonian.

For one alloy configuration, the retarded propagator $\mathcal{G}^{\mathrm{r}}\left(t, t^{\prime}\right)$ is simply the evolution operator of $H(t)$ multiplied by $i \hbar v\left(t-t^{\prime}\right)$. For the configuration averaged quantities we will use italics, $G^{\mathbf{r}}=\left\langle\mathcal{G}^{\mathrm{r}}\right\rangle$, etc. To obtain $G^{\mathrm{r}}$, we configuration a verage the equation of motion for $\mathcal{G}^{\mathrm{r}}$, introduce the self-energy and get the Dyson equation

$$
\mathrm{i} \hbar \partial_{t} G^{\mathrm{r}}\left(t, t^{\prime}\right)-\langle H(t)\rangle G^{\mathrm{r}}\left(t, t^{\prime}\right)-\int \mathrm{d} \bar{t} \Sigma^{\mathrm{r}}(t, \bar{t}) G^{\mathrm{r}}\left(\bar{t}, t^{\prime}\right)=\delta\left(t-t^{\prime}\right) .
$$

To obtain a closed equation of motion, we express $\Sigma$ as an approximate functional of $G$ using the coherent potential approximation (CPA). In the CPA, the self-energy $\Sigma$ is decomposed into single site contributions which are non-random. On a single site, the true $\mathcal{D}_{i}$ is substituted back for $\Sigma_{i}$; this local disturbance is characterized by a single site scattering matrix $\mathcal{T}_{i}$. The Soven condition [5] requires that this scattering vanish on average

$$
\left\langle\mathcal{T}_{i}\right\rangle=0
$$

This is an implicit equation for $\Sigma$. 


\section{Adiabatic approximation for the self-energy}

For a general time dependence of the external field $\mathcal{E}(t)$, the Dyson equation is prohibitively difficult for an explicit solution, as the double time structures of Eq. (4) do not lead to convolutions. On top of that, the self-energy is not known beforehand. This is apparent in the weak scattering limit of the CPA ("self-consistent Born approximation"):

$$
\Sigma_{i}^{\mathrm{r}}\left(t, t^{\prime}\right)=\left\langle\mathcal{D}_{i} G^{\mathrm{r}}\left(t, t^{\prime}\right) \mathcal{D}_{i}\right\rangle
$$

Thus, in the course of solving the Dyson equation, we have to generate the self-energy by introducing $G$ into the latter equation, or, in the general case, into the Soven condition (5).

Our aim is now to decouple the construction of $\Sigma$ from the solution of the Dyson equation. The procedure, somewhat in the spirit of Ch. 9 of the book by Kadanoff and Baym [6], is based on two observations:

1. For given times $t_{1}>t_{2}$, the GF is determined only by the $\mathcal{E}(t)$ with $t_{1} \geq$ $t \geq t_{2}$. For the alloy GF, this statement is exact, because the unaveraged $\mathrm{GF}$ has a time-local Hamiltonian and the $\langle\cdots\rangle$ is also instantaneous.

2. The self-energy is appreciable only in a finite range of the difference $t-t^{\prime}$, shorter than the typical quasiparticle lifetimes and characterizing, in fact, the quasiparticle formation time $\tau_{\text {FORM }}$. This second statement has only a qualitative character, as the self-energy will usually have weak long time tails connected with the branching singularities of $\Sigma$ in the complex energy plane [7].

With these assumptions, we will introduce an auxiliary self-energy $\bar{\Sigma}\left(t-t^{\prime} \mid \mathcal{E}\right)$ corresponding to, and parametrized by, a steady illumination $\mathcal{E}$. It depends only on the time difference, as indicated. We proceed to our basic approximation

$$
\Sigma\left(t, t^{\prime}\right) \sim \bar{\Sigma}\left(t-t^{\prime} \mid \mathcal{E}(t)\right) \text {. }
$$

The "upper" time appears in the approximate $\Sigma$ at two places. First, as a parameter of the slowly varying external field $\mathcal{E}(t)$. Second, in the time difference, with respect to which the $\Sigma$ changes rapidly. The approximation has an asymptotic nature, with a semi-quantitative criterion

$$
\frac{\left|\mathcal{E}(t)-\mathcal{E}\left(t-\tau_{\mathrm{FORM}}\right)\right|}{\mathcal{E}(t)} \ll 1
$$

The meaning of the approximation can be followed in Eq. (6). If $\mathcal{E}$ does not change much between $t^{\prime}$ and $t$, the GF inside the average can be replaced by its steady illumination approximant. Note that the approximate self-energy obeys the Soven condition exactly. The loss of self-consistency occurs between the approximant GF and the solution of the Dyson equation.

\section{Actual construction of the adiabatic self-energy}

The actual calculation of the adiabatic self-energy is simplified by Fourier transforming $\Sigma$ with respect to $t-t^{\prime}$ and solving the Soven equation (5) in the complex energy plane. Furthermore, for our model (3), the self-energies are specified by a single $c$-function

$$
\Sigma=\sigma P_{c}, \quad \bar{\Sigma}=\bar{\sigma} P_{c} .
$$


With this, and using the definitions (1)-(3), it is easy to obtain the Soven equation in an explicit form. We write it here for the instructive case (6):

$$
\bar{\sigma}(z)=\left\langle(\epsilon-\langle\epsilon\rangle)^{2}\right\rangle \sum_{k \in \mathrm{BZ}} \frac{1}{z-\epsilon_{\mathrm{c}}-\langle\epsilon\rangle-\bar{\sigma}(t)-|Q|^{2} /\left(z-\epsilon_{\mathrm{v}}-\hbar \omega\right)} .
$$

IIere, $\epsilon_{\mathrm{c}}$ and $\epsilon_{\mathrm{v}}$ are $k$-dependent dispersion laws. Up to the secondary renormalization effects, the modifications of the self-energy caused by the light concentrate near the crossover of $\epsilon_{\mathrm{c}}-\langle\epsilon\rangle$ and $\epsilon_{\mathrm{v}}+\hbar \omega$, which is the $k$-point of the weak field vertical transitions. Also $Q=-e \mathcal{E} \cdot x_{\mathrm{cv}}(k)$ is $k$-dependent in principle, but we neglect this, taking for $Q$ the value at the resonant crossover. $Q$ is the hybridization strength, and with no disorder, it would cause a gap in the hybridized bands of the width $2|Q|$. For a constant complex $\bar{\sigma}$, this gap would always be smeared. In the self-consistent treatment, the coherent action of the light and of the disorder leads to a complex behavior which we want to demonstrate presently. $\Sigma$ depends on the
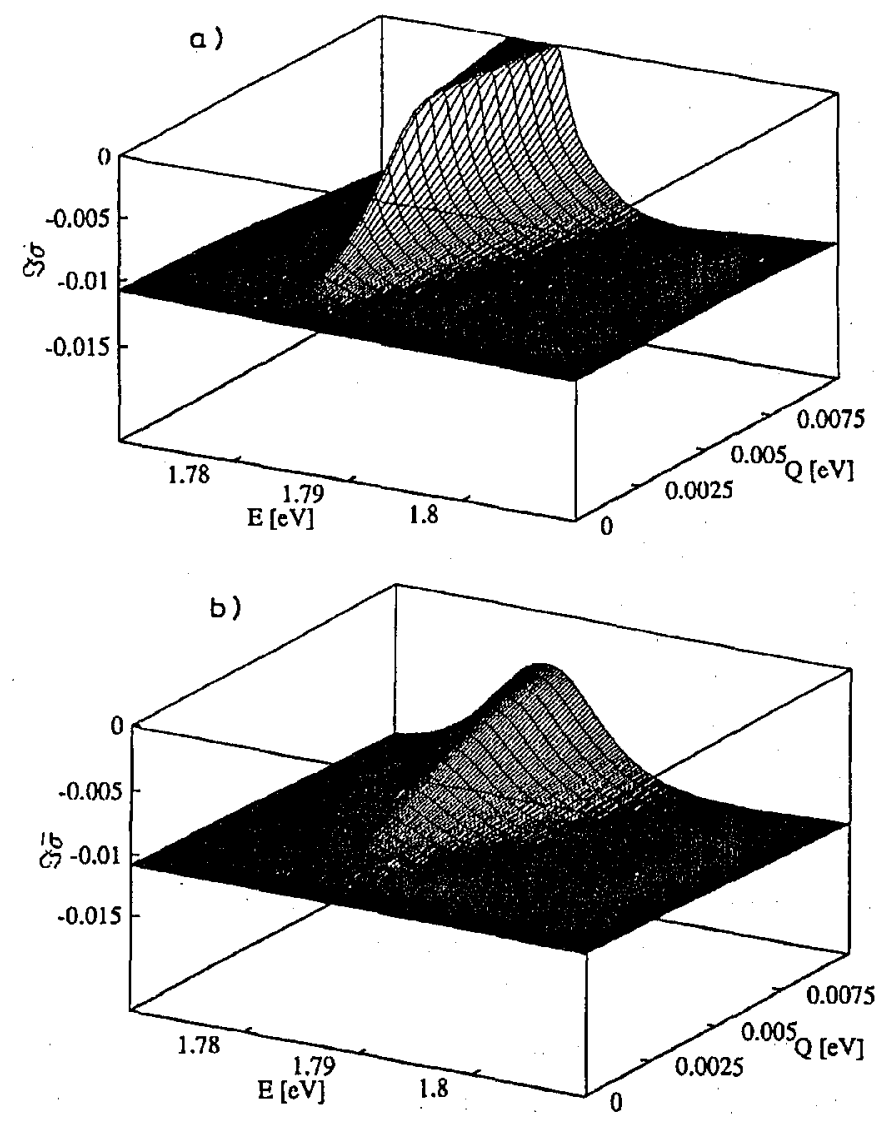

Fig. 1. (a) The retarded GF $\mathcal{I} \bar{\sigma}(E+\mathrm{i} 0, Q)$ as a function of $E$ and $Q$ for given alloy parameters. (b) The same as in Fig. 1a, but with the smearing value $\hbar / 0.3$ ps. 
energy and on two parameters, $Q$ characterizing the illumination, and $\left\langle(\epsilon-\langle\epsilon\rangle)^{2}\right\rangle$ measuring the disorder; the latter in turn depends on the alloy composition and the atomic level displacements. We consider a binary alloy with a moderate disorder leading to quasiparticle lifetimes in the order of $0.1 \mathrm{ps}$ and the values of $Q$ in the same range to make the effect of both ingredients comparable. The estimates show that this corresponds to usual laboratory subpicosecond light pulses.

Figure 1a shows a $Q$-dependence typical of a given alloys, as would correspond to an actual pulse. The quantity shown is $\mathcal{I} \bar{\sigma}(E+\mathrm{i} 0, Q)$. It is negative; the mesa at the zero value represents the optically induced gap. Without disorder, it would close just at $Q=0$. Here we see two regimes; it can be seen that important vestiges of the gap persist also for weaker $Q$, however. It can also be seen that the basic plateau far from the gap is largely determined by the disorder, and its $Q$-dependence is very weak.

Figure $1 \mathrm{~b}$ characterizes the same situation, but with an additional inelastic scattering simulated by a small imaginary part of $E$. We chose the smearing to be $\hbar / 0.3 \mathrm{ps}$, comparable with the alloy scattering effect, and the self-energy behaves correspondingly: certainly the gap effect is not suppressed, at least for stronger illuminations.

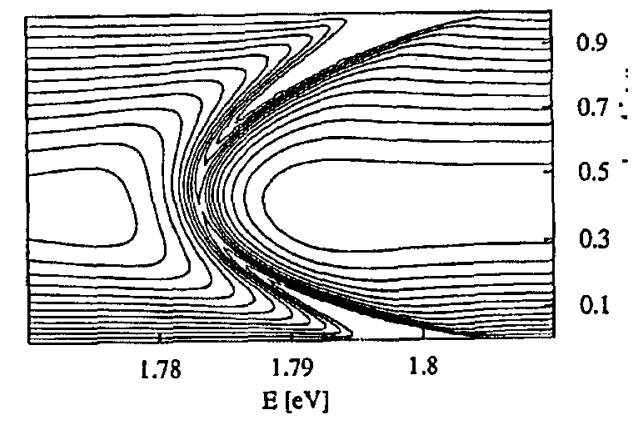

Fig. 2. The retarded GF for constant illumination $(Q=0.005) \mathrm{eV}$, but for alloy concentration varying in the range $0-100 \%$.

Finally, in Fig. 2 the $\mathcal{I} \bar{\sigma}$ is shown again, this time for a constant illumination $Q=0.005 \mathrm{eV}$ but in dependence on the binary alloy composition. A neat "Nordheim" parabolic depedence of the crest of the self-energy landscape can be seen. For smaller concentrations, a gap opens up, but for concentrated alloys it gets closed for our choice of parameters.

To conclude, this type of study permits to classify various situations and to develop a better understanding of the fast excitation processes in semiconductor alloys.

\section{Acknowledgment}

This research was supported by the Grant Agency of the Czech Republic under project number 202/96/0098. 


\section{References}

[1] A. Kalvová, B. Velický, Z. Phys. B 94, 273 (1994).

[2] B. Velický, A. Kalvová, Phys. Status Solidi B 188, 515 (1995).

[3] A. Kalvová, B. Velický, Acta Phys. Pol. A 88, 767 (1995).

[4] V.M. Galitskii, S.P. Goreslavskii, V.F. Elesin, Sov. Phys.-JETP 30, 117 (1970).

[5] A. Gonis, Green Function for Order and Disorder Systems, North-Holland, Amsterdam 1992.

[6] L.P. Kadanoff, G. Baym, Quantum Statistical Mechanics, W.A. Benjamin, New York 1962.

[7] P. Lipavský, F.S. Khan, J.W. Wilkins, Phys. Rev. B 43, 6665 (1991). 